

\title{
Research
}

\section{Incidence and patient demographics of pre-hospital anaphylaxis in Tasmania, Australia}

\author{
Melanie L Blackhall, MPhil, Lecturer ${ }^{1}$, Dale Edwards, MEd, Senior Lecturer ${ }^{1}$
}

Affiliation:

${ }^{1}$ School of Medicine, University of Tasmania, Hobart, Tasmania, Australia

\section{Abstract}

\section{Introduction}

It is widely accepted that anaphylaxis is an increasing problem worldwide. This inquiry into pre-hospital incidence and patient demographics is useful to determine the status of anaphylaxis in Tasmania, Australia. The objective of this study was to determine the incidence of pre-hospital anaphylaxis in Tasmania and identify trends in characteristics of affected patients.

\section{Methods}

Raw data was searched and extracted from Ambulance Tasmania electronic recording system and case records for the period 1 January 2008 to 31 December 2011 following approval from the University of Tasmania's Higher Education Research Council. This involved a chart review of 226,421 cases with the search parameters of anaphylaxis and allergy/allergic reaction.

\section{Results}

There were 1014 patients classified as having an allergic reaction (including the most severe form of anaphylaxis). Of the atopic group, $370(26.7 \%)$ were given a final primary diagnosis of anaphylaxis. The distribution of anaphylaxis cases was higher in adult females: $180(61.6 \%)$ versus $112(38.4 \%)$ in males. Interestingly $21.1 \%$ of the total anaphylaxis cases were paediatric $(n=78)$ with a greater percentage of male $(57.7 \%)$ to female $(42.3 \%)$ cases in the paediatric population. Aetiology was identified in $83.5 \%$ of the cases: envenomation (insects) 131 (42.4\%); food 113 (36.6\%); medication 52 (16.8\%); known other 10 (3.2\%); exercise 3 $(<1 \%)$. Sixty-one patients $(16.5 \%)$ had anaphylaxis from an unknown aetiology. Almost two-thirds $(61.9 \%)$ of the cases were from southern Tasmania.

\section{Conclusion}

These findings suggest that almost a quarter of all calls for allergy or allergic reaction are of a severe potentially life-threatening nature, with the most common aetiology being envenomation from insects (jack jumper bites).

\section{Keywords}

anaphylaxis, pre-hospital, Tasmania

Corresponding Author: Melanie Blackhall, Melanie.Blackhall@utas.edu.au 


\section{Introduction}

Anaphylaxis is a severe life-threatening allergic reaction involving multiple organ systems. The condition is predominantly an Immunoglobulin E ( $\mathrm{gE})$ mediated response causing mast cell degranulation and release of mediators that trigger the associated clinical signs and symptoms. It is considered a type 1 hypersensitivity reaction that occurs in both children and adults. According to the Australian Society of Clinical Immunology and Allergy (ASCIA), in order for the allergic reaction to be classified as anaphylaxis there needs to be involvement of either the cardiovascular and/or the respiratory system (1). Much of the aetiology and treatment protocols are not well understood in the community and this drives the purported dichotomy between clinician and general consumer (2).

It is widely accepted that prevalence rates of anaphylaxis across the United States (US) and United Kingdom (UK) have increased in recent years (3-5). Figures suggest one in every 170 children and one in every 1000 adults are affected (6). While there is some conjecture over the diagnosis of cases impacting prevalence rates, it is thought that underdiagnosis is more common than overdiagnosis (7). In Australia, allergy affects approximately 4.1 million people (19.6\% of the population) (6). The exact figures for anaphylaxis prevalence are less clear. In a sample of over 4000 school-aged children, Boros et al (2) determined the prevalence of parent-reported allergy and anaphylaxis to be $7.3 \%$ and $0.59 \%$ respectively. It is estimated that anaphylaxis affects one in 170 children (2) in comparison with 30 per 100,000 adults (8).

The consequence of an increase in prevalence is the corresponding financial burden that will accompany it. A report commissioned by ASCIA released in 2007, found the estimated burden of disease to be in excess of $\$ 30$ billion in Australia (9). This included direct costs to the healthcare system in excess of $\$ 1$ billion for the management of anaphylaxis and $\$ 21.3$ billion associated with the indirect morbidity and mortality burden (9).

The importance of quantifying anaphylaxis is to allow insight into the current milieu, as this understanding has the potential to reduce both the financial costs and the resultant burden of disease. Specifically it will inform services, development of appropriate treatment guidelines and programs with the end goal of improving the quality of life for those with the disease. There has been a significant amount of research to date that has centred on hospital presentations, treatment and outcomes of anaphylaxis in the hospital environment (10-19). In addition, there have been a number of investigations into pre-hospital treatment of anaphylaxis. The primary focus of these studies was patient management $(20,21)$ and medication (adrenaline) administration $(22,23)$ with specific focus on the paediatric population $(24,25)$.

Investigation into the current status of anaphylaxis will contribute to a greater understanding of how the disease presents within the community. This is important, as paramedics are often the first line in response and treatment for community presentations of anaphylaxis. Early recognition and management of anaphylaxis has been identified as a key factor in minimising the impact of the disease (26).

The information gleaned from the current investigation will provide depth to the dialogue around the needs of the community in managing anaphylaxis and has the potential to impact morbidity and mortality in the Tasmanian population. In particular, it will provide insight into pre-hospital incidence of anaphylaxis and the disease characteristics, which will be invaluable in regards to how the patient is managed before triage and treatment in the emergency department.

The objective of this study was to determine the incidence of allergic and anaphylactic reactions as diagnosed by paramedics in Tasmania (Australia), and to identify trends in the characteristics of the affected patients, by using a retrospective chart review.

\section{Methods}

\section{Study Design}

The study design was a retrospective analysis of data recorded by Ambulance Tasmania from 1 January 2008 to 31 December 2011.

\section{Setting}

Ambulance Tasmania provides emergency ambulance care and a non-emergency patient transport service through a network of 53 urban, rural and remote ambulance stations across the state, servicing a Tasmanian population of over 510,000 people. Medical retrieval services also utilise fixed and rotary wing aircraft, and transport patients to and from Tasmanian hospitals and other government healthcare facilities (27).

\section{Definitions}

Paediatric cases were identified as those in which the patient was aged less than 16 years at the time of the emergency call. All other cases were deemed adult.

\section{Data collection}

Data were collected from the Victorian Ambulance Clinical Information System (VACIS), which provides a framework for paramedics to collect and report patient data, clinical interventions and patient trends during their pre-hospital treatment. The information collected included the date of the emergency call, the dispatcher's description of the case, the priority level based on the patient's symptoms at the time of the call (1: time critical; 2: acute non-time critical; 3: non-acute), patient demographics (gender, age, location at time of call), the likely aetiology of the reaction, known allergies as well as histories of anaphylaxis and/or asthma. The clinical observations made by the paramedics at the time of their arrival and the final primary assessment (paramedic's diagnosis) was also collected. 


\section{Procedures}

This study focused on patients requesting emergency assistance that were given a final primary assessment of allergy and/or anaphylaxis by the attending paramedic, as determined by paramedic diagnostic criteria. The primary data search was undertaken by Ambulance Tasmania technical staff, who provided VACIS data from all Tasmanian cases between 1 January 2008 and 31 December 2011. All patients given a final primary assessment of allergy and/or anaphylaxis were included in the study. Cases that were non-emergency patient transports or contract cases were excluded from the study. Case records were reviewed and cases in which allergy was unlikely to have been the cause of the reaction, were also excluded from the study.

\section{Outcome measures}

Primary outcome measures related to (a) the number of patients requiring assistance for allergic reactions as compared to the total number of calls to Ambulance Tasmania and (b) the clinical severity of the allergic reaction (triage priority given by the emergency call centre [1,2, or 3], the final primary diagnosis as given by the attending paramedic).

\section{Ethics}

Ethics approval was sought from the University of Tasmania's Higher Education Research Council (HERC). As the data provided by Ambulance Tasmania was de-identified, the research was deemed to be of no significant risk and hence exempt from the formal ethics approval process in accordance with paragraph 5.1.22 of the National Statement on Ethical Conduct in Human Research (2007 - updated 2013) (28).

\section{Results}

Between 1 January 2008 and 31 December 2011, 2149 cases were classified as allergy by the emergency services dispatcher prior to paramedic arrival. Of these, 1,014 were given a final primary assessment by the attending paramedic, with an additional 370 given a final primary diagnosis of anaphylaxis (Table 1). This equates to $26.7 \%$ of allergic reaction classified as potentially life-threatening severe allergic reaction (anaphylaxis).

Table 1. Incidence of anaphylaxis and allergy to total valid incident counts from Ambulance Tasmania per calendar year

\begin{tabular}{|c|c|c|c|c|}
\hline Year & $\begin{array}{c}\text { Ambulance Tasmania } \\
\text { incident count }\end{array}$ & Allergic reaction & Allergy & Anaphylaxis \\
\hline 2008 & 52,639 & 212 & 164 & 48 \\
2009 & 55,688 & 359 & 257 & 102 \\
2010 & 58,425 & 421 & 320 & 101 \\
2011 & 59,669 & 392 & 273 & 119 \\
\hline Total & 226,421 & 1385 & 1014 & 370 \\
\hline
\end{tabular}

Females accounted for more cases of anaphylaxis in adults with $180(61.6 \%)$ of the adult anaphylaxis cases being women (Table 2). This is juxtaposed with paediatric cases of anaphylaxis, which saw boys at a slightly greater risk with 45 $(57.7 \%)$ paediatric cases being male.
Over half of the anaphylaxis cases occurred in the southern region of Tasmania (61.9\%), while northern and north-western regions accounted for $22.4 \%$ and $13.8 \%$ of cases, respectively (Table 3).

Table 2. Patient demographics of cases with final primary assessment of anaphylaxis ( $N=370)$

\begin{tabular}{|c|c|c|c|c|c|}
\hline Year & Total cases & \multicolumn{2}{|c|}{ Adult cases } & \multicolumn{2}{c|}{ Paediatric cases } \\
\hline & & Male & Female & Male & Female \\
\hline 2008 & 48 & 12 & 21 & 10 & 5 \\
2009 & 102 & 29 & 50 & 14 & 9 \\
2010 & 101 & 32 & 54 & 8 & 7 \\
2011 & 119 & 39 & 55 & 13 & 12 \\
\hline Total & 370 & 112 & 180 & 45 & 33 \\
\hline
\end{tabular}


Table 3. Representation of anaphylaxis cases by region

\begin{tabular}{|c|c|c|c|c|c|}
\hline Year & Total cases & South & North & North-west & Unknown \\
\hline 2008 & 48 & 24 & 14 & 8 & 2 \\
2009 & 102 & 73 & 15 & 14 & 0 \\
2010 & 101 & 64 & 20 & 14 & 3 \\
2011 & 119 & 68 & 34 & 15 & 2 \\
\hline Total & 370 & 229 & 83 & 51 & 7 \\
\hline
\end{tabular}

Of the 370 cases, 130 (35.1\%) had a known history of anaphylaxis and 52 (14.1\%) had a known history of asthma (Table 4). Of the patients with a history of anaphylaxis, $6.5 \%$ also had a history of asthma. The aetiology of reactions within the paediatric population showed: $48.7 \%(n=38)$ food, $30.7 \%$ $(n=24)$ envenomation by insect and $17.9 \%(n=14)$ from other or unknown cause (Table 5). Within the adult population, the most common aetiology was envenomation $(36.6 \%, n=107)$, followed by food $(25.7 \%, n=75)$, medication $(17 \%, n=50)$ and unknown causes $(26.8 \%, n=49)$.
Table 4. Prior histories of anaphylaxis and asthma in cases with final primary assessment of anaphylaxis

\begin{tabular}{|l|c|}
\hline \multicolumn{1}{|c|}{ Prior history } & Cases \\
\hline Anaphylaxis & 130 \\
Asthma & 52 \\
Anaphylaxis and asthma & 24 \\
No known allergies & 103 \\
\hline
\end{tabular}

Table 5. Aetiology of cases with final primary assessment of anaphylaxis

\begin{tabular}{|l|c|c|c|}
\hline \multicolumn{1}{|c|}{ Aetiology } & Total cases & Adult cases & Paediatric cases \\
\hline Envenomation & 131 & 107 & 24 \\
Jack jumper & 57 & 45 & 12 \\
Bee & 21 & 16 & 5 \\
Known other insect & 19 & 18 & 1 \\
Unknown insect & 34 & 28 & 6 \\
Food & 113 & 75 & 38 \\
Medication & 52 & 50 & 2 \\
Unknown & 61 & 49 & 12 \\
Known other & 10 & 8 & 2 \\
Exercise & 3 & 3 & 0 \\
\hline \multicolumn{1}{|c|}{ Total } & 370 & 292 & 78 \\
\hline
\end{tabular}

\section{Discussion}

This study examined the incidence of allergic reactions and anaphylaxis in the pre-hospital environment, as diagnosed and managed by paramedics in Tasmania.

This is an essential piece of work as to date statistics for anaphylaxis presentations and prevalence rates have been extrapolated from nationally or internationally reported statistics. The main finding was that during the 4-year study period, 1385 cases were triaged and reported as an allergic reaction, 370 of which had a final primary assessment of anaphylaxis. This equates to $26.7 \%$ of the calls received for allergic conditions being of a severe potentially life-threatening nature.
In addition to the total numbers with a final primary assessment of anaphylaxis, it was identified that there was a higher rate in female (57.6\%) compared to male patients. A regional skew was also identified, with over $60 \%$ of the cases being from the southern Tasmania region. It may be argued that these findings are both a representation of wider population trends and geographical distribution of the population.

Evidence exists that a combination of active asthma and anaphylaxis leads to a higher likelihood of mortality during an anaphylactic reaction, as reported by Liew et al (12). This signals a great degree of concern for poor outcomes in the $6.5 \%$ of patients presenting with the combined risk factors of asthma and anaphylaxis, and calls for increased vigilance and understanding of the complications associated with comorbidities. 
Further to this, there were 78 paediatric cases reported, equating to $21.1 \%$ of the total cases of anaphylaxis in the study period. Within these paediatric cases, the aetiology of the reactions were of interest, with food and insect bite aetiology accounting for $48.7 \%$ and $30.7 \%$ of cases respectively. While evidence from the US supports these findings (7), a recent Australian study found the most common trigger in paediatric population to be food (29) at a reported rate of $85 \%$ of cases (29), much higher than the current study's findings.

The significance of this lies in the potential for harm and the application of appropriate treatment protocols. From an investigation into causes of fatal anaphylaxis in the UK, Pumphrey found respiratory arrest was the major factor in food related anaphylaxis compared with shock in cases where insect venom was the trigger (26). This was further compounded by much shorter median time to death from insect venom versus food (26). The high number of paediatric cases identified in this study suggests a need for specialised paediatric anaphylaxis care, with an emphasis on the timeframe within which treatment is administered to these patients.

\section{Limitations of this study}

A limitation of the research was that the study was retrospective by design. This is a challenge for epidemiological research as researchers are faced with extrapolating data from records that may be absent or incomplete.

The VACIS reporting system for patient records only came online in Tasmania in 2007. As a period of adjustment follows the introduction of any new record-keeping system, this could account for the low reported numbers for anaphylaxis in 2008, which impacted prevalence rates/trends. Extending the search parameters to include more recent years would enable a better prediction of 5-year history for anaphylaxis in Tasmania.

\section{Conclusion}

The significant finding of this study is that over a quarter of the calls received by paramedic services in Tasmania for allergy or allergic reaction are classified as the severe form or anaphylactic. Of further significance was the aetiology of the cases in the paediatric population. $30.7 \%$ of the paediatric population had insect envenomation as the cause, which is novel for this population and requires further investigation.

Practical implications of this research are improvement in characterisation with ideally a nationally accepted standard for characterising anaphylaxis cases versus those with a similar appearance (namely severe allergy). Further implications from research of this nature highlight the need for accurate reporting, which creates difficulties for research of this type.

\section{Conflict of interest}

The authors declare they have no competing interests. Each author of this paper has completed the ICMJE conflict of interest statement.

\section{References}

1. ASCIA. Health professional information paper (HPIP). Anaphylaxis, September 2013. Available at: www.allergy.org. au/health-professionals/hp-information/asthma-and-allergy/ anaphylaxis

2. Boros CA, Kay D, Gold MS. Parent reported allergy and anaphylaxis in 4173 South Australian children. J Paediatr Child Health 2000;36(1):3640.

3. Decker WW, Campbell RL, Manivannan V, et al. The etiology and incidence of anaphylaxis in Rochester, Minnesota: A report from the Rochester Epidemiology Project. J Allergy Clin Immunol 2008;122(6):1161-5.

4. Liu AH, Jaramillo R, Sicherer SH, et al. National prevalence and risk factors for food allergy and relationship to asthma: results from the national health and nutrition examination survey 2005-2006. ibid. 2010;126(4):798-806.

5. Sheikh A, Hippisley-Cox J, Newton J, Fenty, J. Trends in national incidence, lifetime prevalence and adrenaline prescribing for anaphylaxis in England. J Royal Soc Med 2008;101(3):139-43.

6. Mullins RJ, Cook M, Doublass J, Mallon D, Smith J, Wong M. The economic impact of allergic disease in Australia: not to be sneezed at. ASCIA/Access Economics Report, November 2007. Available at: www.allergy.org.au/images/stories/ pospapers/2007_economic_impact_allergies_report_13nov. pdf

7. Dinakar C. Anaphylaxis in children: current understanding and key issues in diagnosis and treatment. Curr Allergy Asthma Rep 2012;12(6):641-9.

8. Yocum MW, Butterfield JH, Klein JS, et al. Epidemiology of anaphylaxis in Olmsted County: a population-based study. J Allergy Clin Immunol 1999;104:452-6.

9. ASCIA. Allergy and immune diseases in Australia (AIDA) report, July 2013. Available at: www.allergy.org.au/images/ stories/reports/ASCIA_AIDA_Report_2013.pdf

10. Rappo TB, Cottee AM, Ratchford A, Burns BJ. Tick bite anaphylaxis: incidence and management in an Australian emergency department. Emerg Med Australas 2013;25(4):297-301.

11. Gibbs NM, Sadleir PH, Clarke RC, Platt PR. Survival from perioperative anaphylaxis in Western Australia 2000-2009. Br J Anaesth 2013;111(4):589-93.

12. Liew WK, Williamson E, Tang MLK. Anaphylaxis fatalities and admissions in Australia. J Clin Immunol Allergy 2009;123(2):434-42.

13. Sadleir PH, Clarke RC, Bunning DL, Platt PR. Anaphylaxis to neuromuscular blocking drugs: incidence and cross-reactivity in Western Australia from 2002 to 2011. Br J Anaesth 
14. Churchman A1, O'Leary MA, Buckley NA, et al. Clinical effects of red-bellied black snake (Pseudechis porphyriacus) envenoming and correlation with venom concentrations: Australian Snakebite Project (ASP-11). Med J Aust 2010;193(11-12):696-700.

15. Mullins RJ, Dear KBG, Tang MLK. Characteristics of childhood peanut allergy in the Australian Capital Territory, 1995 to 2007. J Allergy Clin Immunol 2009;123(3):689-693.

16. Brown SGA, Franks RW, Baldo BA, Heddle RJ. Prevalence, severity, and natural history of jack jumper ant venom allergy in Tasmania. ibid. 2003;111:187-92.

17. Brown SGA, Mullins RJ, Gold MS. Anaphylaxis: diagnosis and management. Med J Aust 2006;185(5):283-89.

18. Mullins RJ, Clark S, Camargo CA Jr. Socio-economic status, geographic remoteness and childhood food allergy and anaphylaxis in Australia. Clin Exp Allergy 2010;40(10):152332.

19. Australian Institute of Health and Welfare. Allergic rhinitis ('hay fever') in Australia. Cat. no. ACM 23. Canberra: AlHW, 2011.

20. Chung T, et al. Pre-hospital management of anaphylaxis in one Canadian Urban Centre. Resuscitation 2014; in press.

21. Capps JA, Sharma V, Arkwright PD. Prevalence, outcome and pre-hospital management of anaphylaxis by first aiders and paramedical ambulance staff in Manchester, UK. Resuscitation 2010;81(6):653-7.

22. Rea TD, Edwards C, Murray JA, Cloyd DJ, Eisenberg MS.
Epinephrine use by emergency medical technicians for presumed anaphylaxis. Prehosp Emerg Care 2004;8(4):40510.

23. Safdar B, Cone DC, Pham KT. Subcutaneous epeinphrine in the prehospital setting. ibid. 2001;5(2):200-7.

24. Lammers R, Willoughby-Byrwa M, Fales W. Medication errors in prehospital management of simulated pediatric anaphylaxis. ibid. 2014;18(2):295-304.

25. Tiyyagura GK, Arnold L, Cone DC, Langhan M. Pediatric anaphylaxis management in the prehospital setting. ibid. 2014;18(1):46-51.

26. Pumphrey RS. Lessons from management of anaphylaxis from a study of fatal reactions. Clin Exp Allergy 2000;30(8):1144-50.

27. Department of Health and Human Services. Service files Ambulance Tasmania, 2014. Available at: www.dhhs.tas.gov. au/service_information/services_files/tasmanian_ambulance_ service [Accessed 28 May 2014].

28. National Health and Medical Research Council 2013. National Statement on Ethical Conduct in Human Research. Commonwealth of Australia. Available at: www.nhmrc.gov. au/_files_nhmrc/publications/attachments/e72_national_ statement_140130.pdf

29. de Silva IL, Mehr SS, Tey D, Tang MLK. Paediatric anaphylaxis: a 5 year retrospective review. Allergy 2008;63(8):1071-6. 